\title{
Physical Non-Contact Communication between Microscopic Aquatic Species: Novel Experimental Evidences for an Interspecies Information Exchange
}

\author{
Daniel Fels \\ University of Basel, Botanical Institute, Hebelstrasse 1, 4056 Basel, Switzerland \\ Correspondence should be addressed to Daniel Fels; daniel.fels@unibas.ch
}

Received 27 October 2015; Revised 31 January 2016; Accepted 11 February 2016

Academic Editor: Jianwei Shuai

Copyright @ 2016 Daniel Fels. This is an open access article distributed under the Creative Commons Attribution License, which permits unrestricted use, distribution, and reproduction in any medium, provided the original work is properly cited.

Previous experiments on physical non-contact communication within same species gave rise to test for this type of communication also across the species border, which was the aim of the present study. It was found that autotrophic unicellular organisms (Euglena viridis), separated by cuvettes, affected the proliferation rate of heterotrophic unicellular organisms (Paramecium caudatum). Further, the heterotrophic unicellular organism affected also the proliferation rate of a multicellular heterotrophic organism (Rotatoria sp.) and vice versa. In the case when populations (of Euglena viridis and Paramecium caudatum) were shielded against electromagnetic fields in the optical spectrum from each other, no effects were measured. The results may support the notion that the organisation of ecosystems relies also on the exchange of electromagnetic fields from their constituting biosystems.

\section{Introduction}

Functional coordination between cells (or unicellular organisms) is mediated by complex processes of information exchange. The carriers of this communication are generally chemical (i.e., neurotransmitters and autoinducer molecules) or physical (e.g., electrical signals) in nature. Despite the well-known chemical information exchange in a non-contact mode between cells, there are experimental evidences starting almost hundred years ago that there is also a physical (i.e., nonchemical) non-contact communication taking place between cells [1]. Pioneering researchers in this regard were the Russian scientists A. Gurvitsch and V. P. Kaznacheev who concluded that there could be an optical (i.e., electromagnetic) cell-to-cell communication (for reviews about this topic see [2-4]).

In order to test these claims with novel modern experiments (e.g., [5, 6]), the present author conducted experiments with Paramecium caudatum populations and could show that indeed intercellular non-contact and nonchemical interaction between the Paramecium caudatum populations could be detected when separated with a glass barrier (or either quartz and normal glass) $[7,8]$. The characteristics of effects were dependent on the type of glass used and numbers of cells involved.

These results motivated the author to hypothesize that a physical non-contact communication (possible via electromagnetic radiation) may have an important role in cellinteractions also within ecological systems (see also [9]). In particular, it was hypothesized that there is a non-contact electromagnetic interaction that is taking place not only between the same species but also between different ones. Such effects were indeed already reported, for example, from bacteria on egg cells of sea urchins or from onion root cells on frog egg cells (e.g., $[10,11])$ but were not repeated with modern experimental setups yet. In order to continue this research, the aim of the present study was to investigate if there is such a non-contact and nonchemical interaction between three different aquatic unicellular eukaryotic species (Euglena viridis, Paramecium caudatum, and Rotatoria sp.).

\section{Materials and Methods}

The experiments were performed using cuvettes of two sizes, where the smaller $(15 \mathrm{~mm} \times 15 \mathrm{~mm} \times 45 \mathrm{~mm})$ could be placed into the bigger $(23 \mathrm{~mm} \times 23 \mathrm{~mm} \times 40 \mathrm{~mm})$. This separates 
populations chemically but not physically, in particular electromagnetically $[7,12]$. The cuvettes consisted in either quartz or glass, which latter could serve as a filter for UV-B and UV-C and may therefore give different results as compared to separation with quartz [7].

The pairs of cuvettes (i.e., small cuvettes placed in the larger ones) containing controls (in one case with graphite shielding) and treatment were randomly placed in a grid where the pairs were at close vicinity but separated from each other by light-barriers.

All populations, that is, inducer and tester (receiver) populations, were kept in a light-safe box during mutual exposure. Exposure lasted $48 \mathrm{hrs}$ with the light-safe box standing in an incubator at $24^{\circ} \mathrm{C}$ (but $22^{\circ} \mathrm{C}$ in experiment $1 \mathrm{~b}$ ). At the end of an experiment the number of individuals in tester populations was counted and used for data analysis. Sizes of tester populations were counted with the help of a binocular microscope.

The organisms were Paramecium caudatum, a unicellular ciliate of about $250 \mu \mathrm{m}$ length, Euglena viridis, a unicellular flagellate of about $60 \mu \mathrm{m}$ length, and Rotatoria sp., a multicellular organism of about $500 \mu \mathrm{m}$ length. All of them inhabit ponds or lakes being potential conspecifics. Paramecia belong to the author's own cultures and were fed with bacteria (for more information please refer to [7]). The photosynthetic Euglena came from a lab stock and was kept in standard mineral water. Rotatoria were originally taken from a contaminated protozoan culture in the lab. They were fed like Paramecia with bacteria.

\subsection{Experiment 1}

2.1.1. Experiment 1a: Interspecies Communication between Paramecia and Euglena. This experiment tested for an effect of Euglena viridis on Paramecia caudatum. The big cuvettes (BC) contained within $1 \mathrm{~mL}$ water about 100,000 Euglena cells. In the small cuvette (SC) there were at the onset of the experiment 100 Paramecia. Controls had just $1 \mathrm{~mL}$ of mineral water in the BC. Paramecia were in their own medium (see [7]). The cuvette pairs consisted either in glass or in quartz. An experimental block consisted in five replicates of each treatment group and was repeated three times. The assessment was the cell division rate, that is, the population size of Paramecia at the end of the exposure time.

2.1.2. Experiment 1b: Interspecies Communication between Paramecia and Euglena with Graphite Shielding. Shielding is a commonly used method when looking for electromagnetic effects between organisms or cells (e.g., $[5,13,14])$. If the signal coming from Euglena and affecting Paramecium caudatum is electromagnetic, then a thin layer of colloidal graphite around the inner cuvette should prevent electromagnetic signals [15] coming from the outer inducer population that could induce an effect on the inner tester population. Using purest colloidal graphite in solution (CRAMOLIN ${ }^{\circledR}$ GRAPHIT) a graphite-layer was twice sprayed onto the bottom and up to a height of $15 \mathrm{~mm}$ on the outer side of the small cuvettes (the volume of $1 \mathrm{~mL}$ reaches in a cuvette pair only $4 \mathrm{~mm}$ in the outer and $6 \mathrm{~mm}$ in the inner cuvette).
TABLE 1: Listed are the mean values and standard errors $(n=15)$ of the absolute population size of Paramecium caudatum at the end of the exposure to Euglena viridis or its absence (yes/no). The separation between the species was conducted with cuvettes made of glass $(\mathrm{G})$ or quartz $(\mathrm{Q})$.

\begin{tabular}{lcc}
\hline Exposure to Euglena & Material & Mean \pm standard error \\
\hline Yes & G & $192.1 \pm 4.2$ \\
Yes & Q & $202.6 \pm 5.1$ \\
No & G & $215.7 \pm 5.2$ \\
No & Q & $213.1 \pm 4.8$ \\
\hline
\end{tabular}

TABle 2: This table refers to the exposure Experiment la with Euglena viridis on Paramecium caudatum and displays the results of an analysis of variance based on final population size. The ANOVA table displays the sum of squares (SS), degrees of freedom (df), Fratio, and the probability of error (prob $>F$ ).

\begin{tabular}{lcccc}
\hline Source & df & SS & $F$-ratio & Prob $>F$ \\
\hline Material $(\mathrm{G} / \mathrm{Q})$ & 1 & 0.006 & 0.731 & 0.3961 \\
Euglena $(\mathrm{y} / \mathrm{n})$ & 1 & 0.104 & 12.566 & $0.0008^{* * *}$ \\
Material $\times$ Euglena & 1 & 0.015 & 1.843 & 0.1800 \\
\hline
\end{tabular}

${ }^{* * *} P<0.001$.

Only quartz cuvettes were used in this variation of Experiment la and the density of Euglena viridis was $250,000 / \mathrm{mL}$. The design was otherwise as described above with (despite the normal control) the additional control where the inner population of Paramecium was shielded with graphite from Euglena in the outer cuvette. The experimental block consisted in five replicates of each of the three treatment groups and was repeated four times.

\subsection{Experiment 2: Interspecies Communication between} Paramecia and Rotatoria. In the BC there were 100 Rotatoria in $1 \mathrm{~mL}$ medium. In the SC there were 100 Paramecia in $1 \mathrm{~mL}$ medium. There were two controls, one for Rotatoria (with medium only in the SC) and one for Paramecia (with medium only in the BC). Separation occurred with both glass and quartz cuvettes. Controls and treatment cuvette pairs were randomly placed in a grid where the pairs were at close vicinity but separated from each other by light-barriers. The assessment was the population size of Paramecia and that of Rotatoria at the end of the exposure time.

2.3. Statistics. Log-transformed data of population sizes of Rotatoria and Paramecia were used to perform an analysis of variance (ANOVA).

\section{Results}

\subsection{Experiment 1}

3.1.1. Experiment 1a: Interspecies Communication between Paramecia and Euglena. The presence of Euglena cells had a significant effect on growth performance in Paramecia: they displayed a retarded cell division rate when neighboured by Euglena (Tables 1 and 2 and Figure 1(a)). Otherwise there were no effects coming from repeating the experiment (statistics 


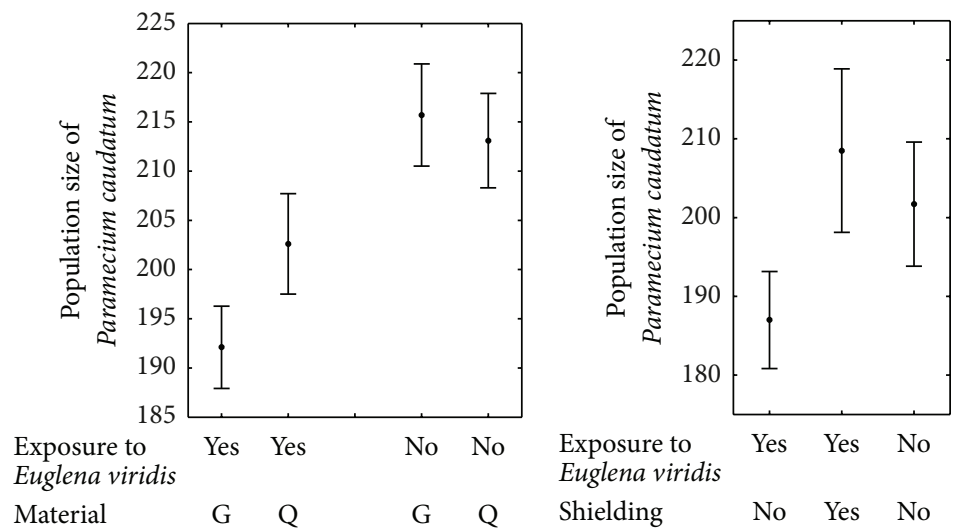

(a) (b)

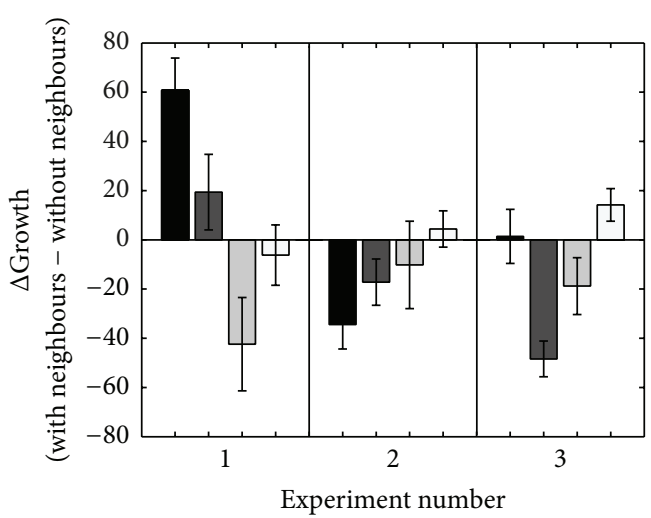

Quartz: Paramecia Glass: Paramecia Quartz: Rotatoria $\square$ Glass: Rotatoria

(c)

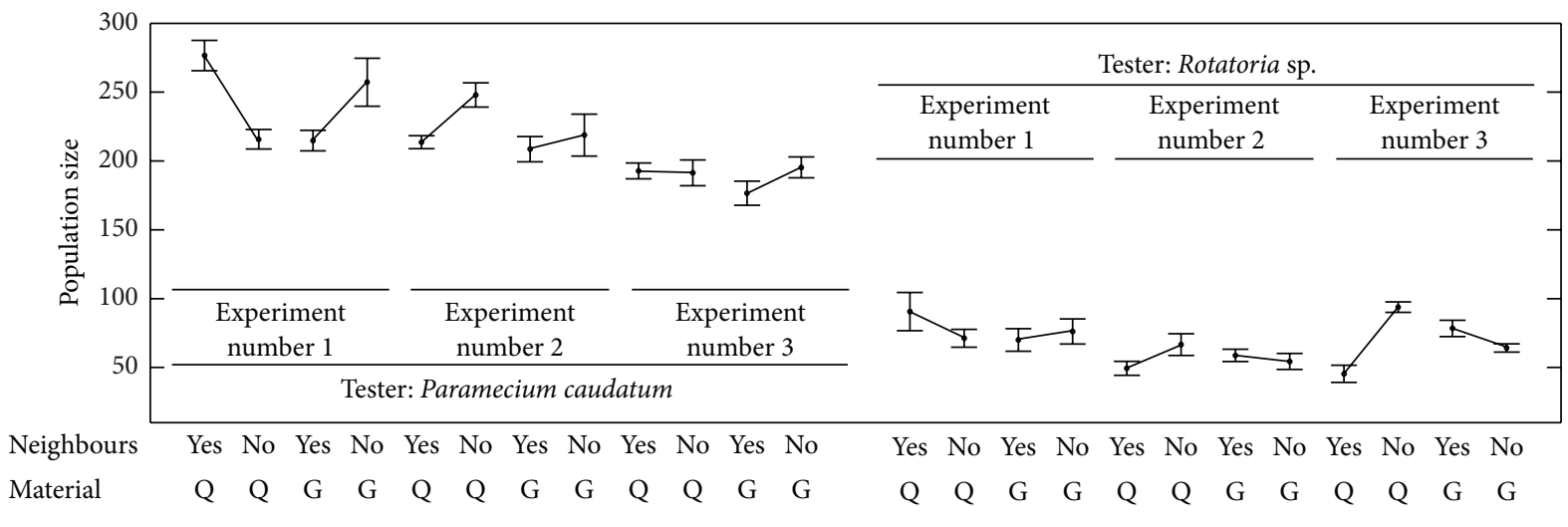

(d)

FIGURE 1: (a-d) represent graphically the results from experiments la and $1 \mathrm{~b}$ and 2. (a) Glass- and quartz-separated populations of Euglena viridis affecting populations of Paramecium caudatum. (b) As in (a) but with graphite shielding and quartz-separation only. (c) refers to experiment 2 and displays the effect of Rotatoria sp. on Paramecium caudatum andvice versa. The $x$-axis shows the three repetitions of experimental blocks and the $y$-axis values of growth with inducer species as neighbour minus growth of controls. (d) refers to the same experiment as in (c) but shows the actual growth values of each treatment group for all three repetitions of experimental blocks.

TABLE 3: This table refers to Experiment $1 \mathrm{~b}$ and lists mean values and standard errors $(n=20)$ of the absolute population size of Paramecium caudatum at the end of the exposure to Euglena viridis or its absence (yes/no). The separation between the species was conducted with cuvettes made of quartz and had two types of controls (normal and shielded; see text).

\begin{tabular}{lcc}
\hline Exposure to Euglena & Shielding & Mean \pm standard error \\
\hline Yes & No & $187.0 \pm 6.2$ \\
Yes & Yes & $208.5 \pm 10.4$ \\
No & No & $201.7 \pm 7.9$ \\
\hline
\end{tabular}

not shown) or from using different separating material (Table 2). After $48 \mathrm{hrs}$ of exposure the BC with Euglena showed green fogy stripes in the medium, most probably chloroplasts that are known to be expulsed under conditions of darkness.
TABLE 4: This table refers to the exposure Experiment 1b (graphite shielding; see text) with Euglena viridis on Paramecium caudatum and displays the results of an analysis of variance based on final population size. The ANOVA table displays the sum of squares (SS), degrees of freedom (df), the $F$-ratio, and the probability of error $($ prob $>F)$.

\begin{tabular}{lcccc}
\hline Source & df & SS & $F$-ratio & Prob $>F$ \\
\hline Repeating the experiment $(\mathrm{RE})$ & 1 & 1.256 & 38.727 & $<0.0001^{* * * *}$ \\
Euglena $(\mathrm{y} / \mathrm{n})$ & 1 & 0.127 & 5.862 & $0.0053^{* *}$ \\
$\mathrm{RE} \times$ Euglena & 1 & 0.063 & 0.966 & 0.459 \\
\hline${ }^{* *} P<0.01,{ }^{* * * *} P<0.0001$. & & & &
\end{tabular}

3.1.2. Experiment 1b: Interspecies Communication between Paramecia and Euglena with Graphite Shielding. The presence of Euglena cells had the same growth retarding effect as in Experiment 1 (Tables 3 and 4 and Figure 1(b)). There were 
TABLE 5: This table shows the mean values and standard errors (SE) for each treatment group, that is, for Paramecium caudatum with or without Rotatoria sp. as neighbours as well as for Rotatoria sp. with or without Paramecium caudatum as neighbours.

\begin{tabular}{|c|c|c|c|c|}
\hline \multirow{3}{*}{ Tester } & \multirow{3}{*}{ Experiment } & \multirow{3}{*}{ Material } & \multicolumn{2}{|c|}{ Neighbours } \\
\hline & & & Yes & No \\
\hline & & & Mean \pm SE & Mean \pm SE \\
\hline \multirow{6}{*}{ Paramecium caudatum } & \multirow{2}{*}{1} & Quartz & $276.6 \pm 11.0$ & $215.8 \pm 7.1$ \\
\hline & & Glass & $214.8 \pm 7.5$ & $257.2 \pm 17.4$ \\
\hline & \multirow{2}{*}{2} & Quartz & $213.6 \pm 4.7$ & $248.0 \pm 8.8$ \\
\hline & & Glass & $208.6 \pm 9.2$ & $218.8 \pm 15.2$ \\
\hline & \multirow{2}{*}{3} & Quartz & $192.8 \pm 5.7$ & $191.4 \pm 9.4$ \\
\hline & & Glass & $176.6 \pm 8.7$ & $195.4 \pm 7.6$ \\
\hline \multirow{6}{*}{ Rotatoria sp. } & \multirow{2}{*}{1} & Quartz & $90.6 \pm 13.9$ & $71.2 \pm 6.5$ \\
\hline & & Glass & $70.0 \pm 8.2$ & $76.2 \pm 9.1$ \\
\hline & \multirow{2}{*}{2} & Quartz & $49.4 \pm 5.1$ & $66.6 \pm 7.9$ \\
\hline & & Glass & $58.8 \pm 4.5$ & $54.4 \pm 5.8$ \\
\hline & \multirow{2}{*}{3} & Quartz & $45.4 \pm 6.2$ & $93.8 \pm 3.8$ \\
\hline & & Glass & $78.4 \pm 5.9$ & $64.2 \pm 3.0$ \\
\hline
\end{tabular}

TABLE 6: This table shows the results from an analysis of variance coming from the experiment on mutual effects of Paramecium caudatum and Rotatoria sp. on each other. The ANOVA table displays the sum of squares (SS), degrees of freedom (df), the $F$-ratio, and the probability of error (prob $>F$ ) based on final population size. Effects came from repeating the experiment (exp), the inducer species (ind), and interactions between repeating the experiment and inducer species $(\exp \times$ ind $)$.

\begin{tabular}{|c|c|c|c|c|c|c|}
\hline Tester & Material & Source & $\mathrm{df}$ & SS & $F$-ratio & prob $>F$ \\
\hline \multirow{6}{*}{ Paramecium caudatum } & \multirow{3}{*}{ Quartz } & $\exp$ & 2 & 0.302 & 22.677 & $<0.0001^{* * * *}$ \\
\hline & & ind & 1 & 0.008 & 1.134 & 0.2975 \\
\hline & & $\exp \times$ ind & 2 & 0.183 & 13.728 & $0.0001^{* * *}$ \\
\hline & \multirow{3}{*}{ Glass } & $\exp$ & 2 & 0.276 & 10.163 & $0.0006^{* * *}$ \\
\hline & & ind & 1 & 0.085 & 6.237 & $0.0198^{*}$ \\
\hline & & $\exp \times$ ind & 2 & 0.022 & 0.810 & 0.4565 \\
\hline \multirow{6}{*}{ Rotatoria sp. } & \multirow{3}{*}{ Quartz } & $\exp$ & 2 & 0.559 & 4.347 & $0.0245^{*}$ \\
\hline & & ind & 1 & 0.585 & 9.090 & $0.0060^{* *}$ \\
\hline & & $\exp \times$ ind & 2 & 1.166 & 9.062 & 0.0012 \\
\hline & \multirow{3}{*}{ Glass } & $\exp$ & 2 & 0.393 & 4.548 & $0.0211^{*}$ \\
\hline & & ind & 1 & 0.034 & 0.782 & 0.3852 \\
\hline & & $\exp \times$ ind & 2 & 0.096 & 1.111 & 0.3456 \\
\hline
\end{tabular}

${ }^{*} P<0.05,{ }^{* *} P<0.01,{ }^{* * *} P<0.001,{ }^{* * * *} P<0.0001$.

also effects coming from repeating the experiment (Table 4). The shielded Paramecium population grew as well as the control population that had no neighbouring inducer population (a contrast analysis showed no difference in growth performance between the two controls: $p>0.241$ ). There was no indication for expulsed chloroplasts.

3.2. Experiment 2: Interspecies Communication between Paramecia and Rotatoria. As there were no material effects but material-neighbour interactions (statistics not shown) the data (Table 5) and their analysis was performed with separating them by material (Table 6). The multicellular Rotatoria sp.and the unicellular Paramecium caudatum have significant effects on each other. In one experimental replication and when separated by quartz the effect was enhancing; otherwise both organisms rather had a reducing effect on the others' proliferation rate (Table 6 and Figures $1(\mathrm{c})$ and $1(\mathrm{~d})$ ).

\section{Discussion}

The results deliver three observations of non-contact and non-chemically induced effects across the species border of two unicellular and one multicellular aquatic organism. The effects, some of which being highly significant, consisted mainly in decreasing the other species' proliferation rate.

It is assumed that the effects were physically transmitted since populations were separated with barriers that disable solvable chemicals from trespassing. Further, volatile substances would not make evolutionary sense to an aquatic organism, and, if existing, any volatile substance would need 
to solve back into the right cuvette. This latter is extremely improbable as the cuvette pairs were very close to each other and, furthermore, treatment groups and controls were randomly placed in a side-by-side manner. In addition, those treatment groups where the inner populations (of Paramecium) were shielded with graphite from the outer population (of Euglena) showed no effect on the tester population giving two important indications. First, if these populations would really use volatiles that would solve back within the same pair of cuvettes, then this should also happen for the treatment group with a graphite shield, which nonetheless is not supported by the data. Second and this was the major reason for the shielding control, as graphite shields electromagnetic waves and no effect from the inducer population was found, this delivers indirect evidence for an electromagnetic signal inducing observed effects.

As the experiments had taken place in total darkness and such darkness does not exist under natural conditions, the effects between the organisms may not have resulted from natural selection. They may, nonetheless, reflect the use of a universal physical code of life.

It is concluded that between the three different species there has been a physical communication. As discussed elsewhere and as graphite shielding gave indirect evidence, it is assumed that this physical communication is of electromagnetic nature [1] implying that theories on electromagnetic (despite chemical) organisation of whole ecosystems may put us on the right track.

\section{Conflict of Interests}

The author declares that there is no conflict of interests regarding the publication of this paper.

\section{Acknowledgments}

The author thanks Felix Scholkmann and an anonymous reviewer for valuable comments on a first version of the paper.

\section{References}

[1] D. Fels, M. Cifra, and F. Scholkmann, Eds., Fields of the Cell, Research Signpost, Trivandrum, India, 2015.

[2] M. Cifra, J. Z. Fields, and A. Farhadi, "Electromagnetic cellular interactions," Progress in Biophysics and Molecular Biology, vol. 105, no. 3, pp. 223-246, 2011.

[3] F. Scholkmann, D. Fels, and M. Cifra, "Non-chemical and noncontact cell-to-cell communication: a short review," American Journal of Translational Research, vol. 5, no. 6, pp. 586-593, 2014.

[4] I. Volodyaev and L. V. Beloussov, "Revisiting the mitogenetic effect of ultra-weak photon emission," Frontiers in Physiology, vol. 6, article 241, 2015.

[5] L. F. Jaffe, "Marine plants may polarize remote Fucus eggs via luminescence," Luminescence, vol. 20, no. 6, pp. 414-418, 2005.

[6] F. Musumeci, A. Scordino, A. Triglia, G. Blandino, and I. Milazzo, "Intercellular communication during yeast cell growth," Europhysics Letters, vol. 47, no. 6, pp. 736-742, 1999.

[7] D. Fels, "Cellular communication through light," PLoS ONE, vol. 4, no. 4, Article ID e5086, 2009.
[8] D. Fels, "Analogy between quantum and cell relations," Axiomathes, vol. 22, no. 4, pp. 509-520, 2012.

[9] L. Brizhik, "On the role and impact of electromagnetic fields in ecosystems," International Journal of Design and Nature and Ecodynamics, vol. 6, no. 4, pp. 272-281, 2011.

[10] N. Choucrun, "On the hypothesis of mitogenetic radiation," Journal of the Marine Biological Association of the United Kingdom, vol. 17, no. 1, pp. 65-74, 1930.

[11] T. Reiter and D. Gabor, Zellteilung und Strahlung, Springer, Berlin, Germany, 1928

[12] D. Fels, "Electromagnetic cell communication and the barrier method," in Fields of the Cell, D. Fels, M. Cifra, and F. Scholkmann, Eds., pp. 149-162, Research Signpost, Trivandrum, India, 2015.

[13] G. Albrecht-Buehler, "Rudimentary form of cellular 'vision,"' Proceedings of the National Academy of Sciences of the United States of America, vol. 89, no. 17, pp. 8288-8292, 1992.

[14] X. Shen, W. Mei, and X. Xu, "Activation of neutrophils by a chemically separated but optically coupled neutrophil population undergoing respiratory burst," Experientia, vol. 50, no. 10, pp. 963-968, 1994.

[15] D. D. L. Chung, "Electromagnetic interference shielding effectiveness of carbon materials," Carbon, vol. 39, no. 2, pp. 279-285, 2001. 

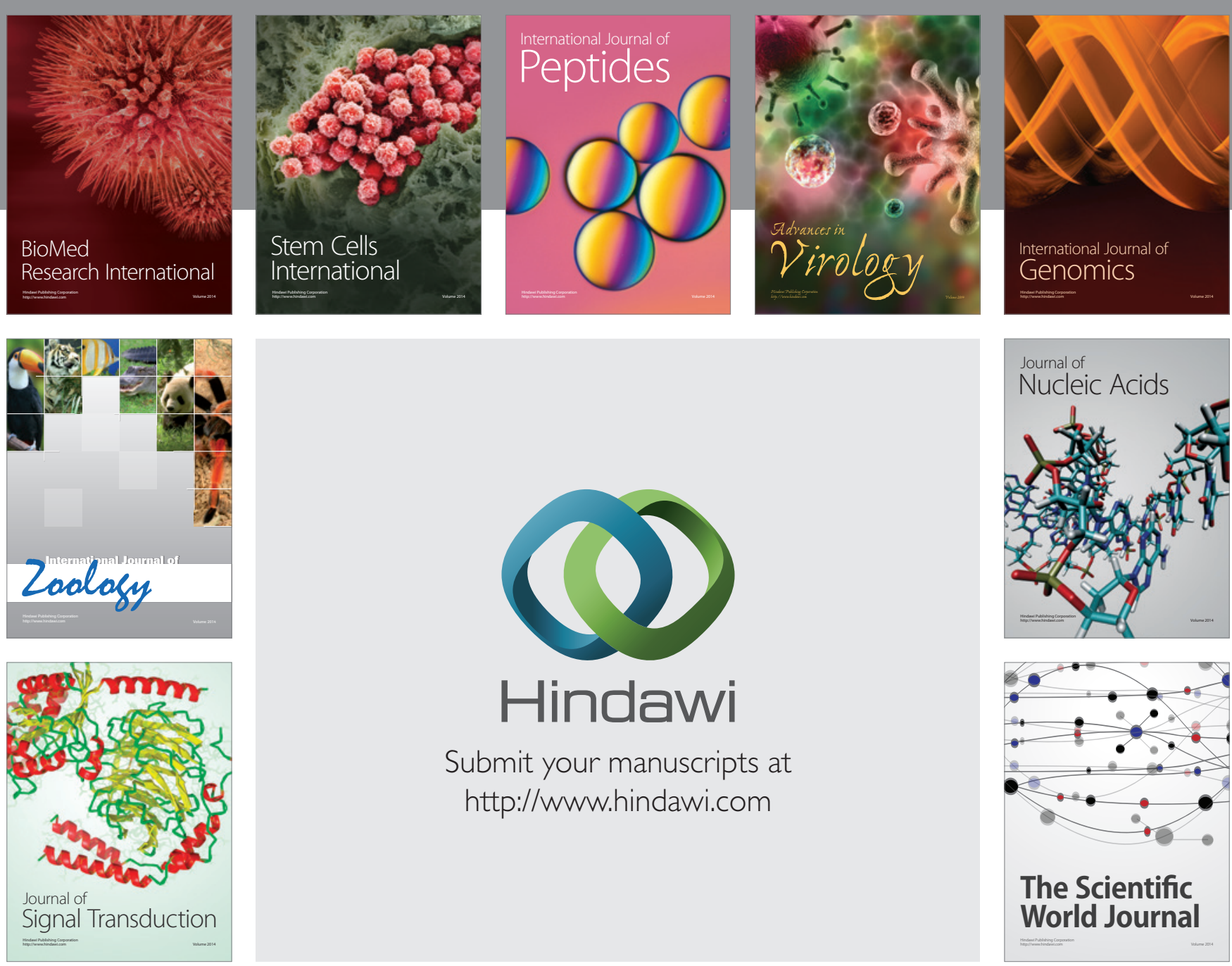

Submit your manuscripts at

http://www.hindawi.com
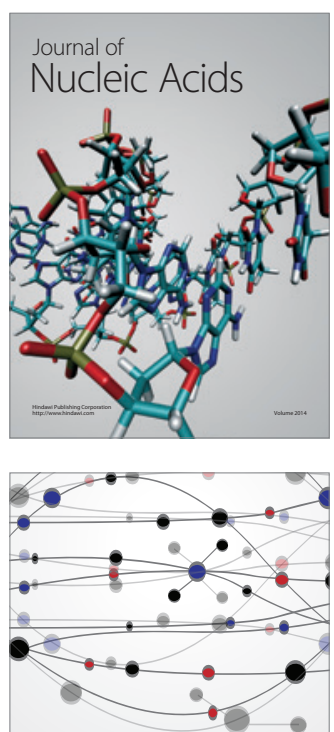

The Scientific World Journal
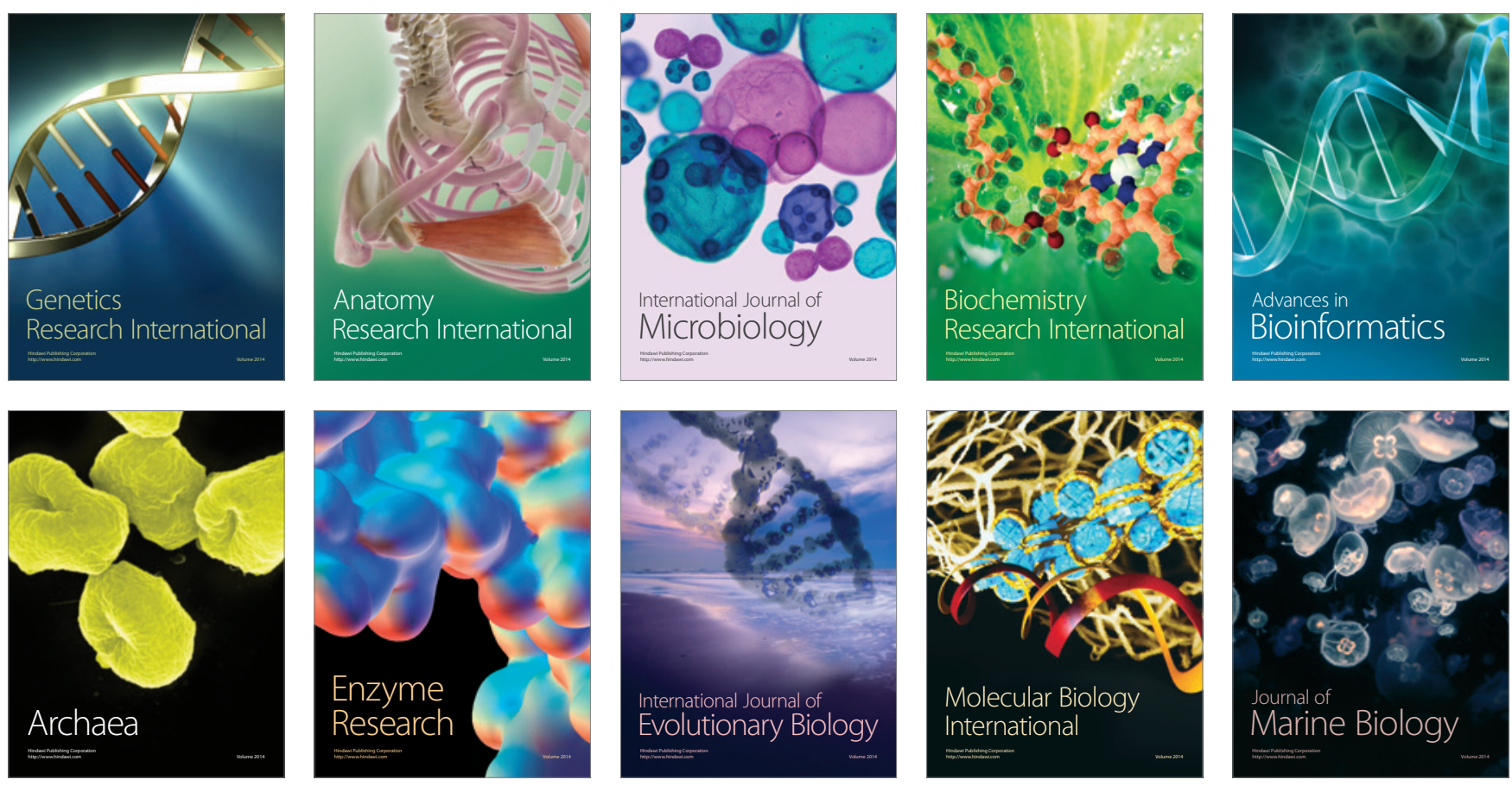